\title{
Electron impact excitation of Fe XVI: radiative and excitation rates ${ }^{\star}$
}

\author{
K. M. Aggarwal and F. P. Keenan
}

Department of Physics and Astronomy, Queen's University, Belfast BT7 1NN, Northern Ireland, UK

e-mail: K.Aggarwal@qub.ac.uk

Received 13 December 2005 / Accepted 16 January 2006

\section{ABSTRACT}

Aims. In this paper we report calculations for energy levels, radiative rates, collision strengths, and effective collision strengths for transitions in Fe XVI.

Methods. For energy levels and radiative rates we have used the General purpose Relativistic Atomic Structure Package (GRASP), and for the compuations of collision strengths the Dirac Atomic $R$-matrix Code (DARC) has been adopted.

Results. Energies for the lowest 39 levels among the $n \leq 7(\ell \leq 4)$ configurations of Fe XVI are reported. Additionally, radiative rates, oscillator strengths, and line strengths are reported for all electric dipole (E1), magnetic dipole (M1), electric quadrupole (E2), and magnetic quadrupole (M2) transitions among these levels. Electron impact excitation collision strengths have also been calculated for all 741 transitions among the above 39 levels over a wide energy range up to 220 Ryd. Resonances have been resolved in the threshold region, and effective collision strengths have been obtained over a wide temperature range up to $10^{7} \mathrm{~K}$. Comparisons are made with the available results in the literature, and the accuracy of the present results is assessed.

Key words. atomic data - atomic processes

\section{Introduction}

This paper is a continuation of our work on generating atomic data (energy levels, radiative rates, collision strengths and excitation rates) for iron ions, for which we have already reported calculations for Fe X (Aggarwal \& Keenan 2004b, 2005b), Fe XI (Aggarwal \& Keenan 2003a,b), Fe XIII (Aggarwal \& Keenan 2004a, 2005a), Fe XV (Aggarwal et al. 2001, 2003a), Fe XVII (Aggarwal et al. 2003b, 2004), Fe XVIII (Jonauskas et al. 2004), Fe XXI (Aggarwal \& Keenan 1999, 2001) and Fe XXIV (McKeown et al. 2004). Here we report similar results for transitions in Fe XVI.

Iron is an abundant element in solar and fusion plasmas, and its emission lines are observed over almost all ionization stages. To analyse the vast amount of observational data available from space missions such as SOHO, Chandra and $\mathrm{XMM}-N e w t o n$, theoretical atomic data for Fe ions are required, as there is a paucity of desired experimental results.

Emission lines of Na-like Fe XVI have been observed in astrophysical plasmas by Behring et al. (1976), Sandlin et al. (1976), Dere (1978), Thomas \& Neupert (1994), and Young et al. (1998). Many of the observed lines have been helpful in

* Tables 2-5 are only available in electronic form at the CDS via anonymous ftp to cdsarc.u-strasbg.fr $(130.79 .128 .5)$ or via http://cdsweb.u-strasbg.fr/cgi-bin/qcat?J/A+A/450/1249 plasma diagnostics (Keenan et al. 1994), the detection of velocity field in the low solar corona (Neupert et al. 1992), and in the interpretation of coronal magnetic field structure (Brosius et al. 1993, 1997). Therefore, a few calculations have been performed in the past, the most notable being those of Tayal (1994), Cornille et al. (1997) and Eissner et al. (1999).

Tayal (1994) calculated results for collision strengths $(\Omega)$ and effective collision strengths $(\Upsilon)$ for transitions among the 10 fine-structure levels of the $\left(1 s^{2} 2 s^{2} 2 p^{6}\right) 3 \ell, 4 s, 4 p$ and $4 d$ configurations. He adopted the CIV3 program of Hibbert (1975) and the standard $R$-matrix code (Berrington et al. 1978) for the calculations of wavefunctions and collision strengths, respectively. In his work, he included configuration interaction (CI) in the generation of wavefunctions, relativistic effects (in a BreitPauli approximation) in calculating values of $\Omega$, and resolved resonances in the thresholds region in order to determine values of $\Upsilon$. However, in the subsequent calculations of Eissner et al. (1999), Tayal's results for $\Omega$, especially for the allowed transitions, have been found to be overestimated - see, for example, Fig. 5 of Eissner et al. This is because in the computations of $\Omega$, he included only a restricted range of partial waves with angular momenta $J \leq 15$, and adopted the procedure of geometric progression to account for the missing higher partial waves. This range of partial waves is clearly insufficient for the convergence of $\Omega$, not only for the allowed but for the forbidden 
transitions as well, as discussed by Eissner et al. Consequently, Tayal's reported results for $\Upsilon$ are also inaccurate (by up to a factor of two) for many transitions, as demonstrated in Fig. 7 of Eissner et al.

Cornille et al. (1997) extended the range of transitions by involving the additional levels of the $4 \mathrm{f}$ and $5 \ell$ configurations, because many of the emission lines involving these levels have already been observed in solar flares (Acton et al. 1985). For their calculations they adopted the SuperStructure (SS) code of Eissner et al. (1974) for the generation of wavefunctions and the distorted-wave (DW) code of Eissner \& Seaton (1972) for the scattering process. They also included relativistic effects through term coupling coefficients, but calculated values of $\Omega$ at energies only above thresholds. Since they did not resolve resonances in the thresholds region, values of $\Upsilon$ estimated from their data of $\Omega$ will be underestimated for many transitions, especially the forbidden ones. Additionally, and more importantly, they included the contribution of partial waves only with $L \leq 25$ up to the highest energy of their calculations, i.e. 200 Ryd. In order to obtain converged results for allowed transitions, they included the contribution of missing higher partial waves through the Coulomb-Bethe approximation, but completely ignored their contribution for the forbidden transitions. As a result of this, their values of $\Omega$ are underestimated by up to $50 \%$, depending on the transition and the energy, as discussed and demonstrated by Eissner et al. (1999).

Finally, Eissner et al. (1999) have performed another calculation for transitions in Fe XVI, for which they adopted the SS program (Eissner et al. 1974) for the generation of wavefunctions, and the standard $R$-matrix program of Berrington et al. (1978) for the computations of $\Omega$. They included 12 finestructure levels among the $3 \ell$ and $4 \ell$ configurations and, as by earlier workers, included CI in the construction of wavefunctions and one-body relativistic effects in determining values of $\Omega$. Additionally, they resolved resonances in the thresholds region, and took particular care to ensure the convergence of $\Omega$ for all types of transitions, and at all energies. Therefore, their reported results for $\Upsilon$ over a wider temperature range up to $10^{7} \mathrm{~K}$ should be comparatively more accurate. However, we find that there is scope for extension as well as improvement over their results, because transitions among the higher excited levels of Fe XVI have already been observed in astrophysical plasmas (see for example, Table 5 of Dere et al. 2001). Therefore, we are extending their calculations up to $39 \mathrm{lev}-$ els which belong to the $n \leq 7$ configurations. By doing so, we also improve upon the results for $\Upsilon$ among the lower levels, because resonances arising from the higher excited levels are now taken into account. Additionally, Eissner et al. reported radiative rates ( $A$-values) for electric dipole (E1) transitions alone, whereas corresponding results for other types of transitions, namely electric quadrupole (E2), magnetic dipole (M1) and magnetic quadrupole (M2), are also required in the modelling of plasmas. Therefore, in this work we report $A$-values for all types of transitions. Finally, our approach is fully relativistic, as we employ the GRASP (General purpose Relativistic Atomic Structure Package) code of Dyall et al. (1989) for the generation of wavefunctions, and the Dirac Atomic $R$-matrix Code
(DARC) of Norrington \& Grant (2006) for computations of $\Omega$, and subsequently of $\Upsilon$.

\section{Energy levels}

The $n \leq 7$ ( $\ell \leq 4)$ configurations of Fe XVI give rise to 39 fine-structure levels, listed in Table 1. Our calculated energies obtained from the GRASP code, with and without including QED effects, are given in this table along with those from the experimental compilations of NIST (http://physics.nist.gov/PhysRefData), and the earlier work of Sampson et al. (1990), which are stored in the CHIANTI database at http://wwwsolar.nrl. navy.mil/chianti.html/. However, energies in CHIANTI are available for the lowest 21 levels only. For our calculations, we have used the option of extended average level (EAL), in which a weighted (proportional to $2 j+1$ ) trace of the Hamiltonian matrix is minimized. This produces a compromise set of orbitals describing closely lying states with moderate accuracy. The inclusion of QED effects lowers the energies by a maximum of 0.015 Ryd. In general, the theoretical energies agree very well, both in magnitude and orderings, with the experimental ones. However, the NIST energy for level $31\left(7 \mathrm{~s}^{2} \mathrm{~S}_{1 / 2}\right)$ is slightly higher by $\sim 0.1 \mathrm{Ryd}$ $(0.3 \%)$. Since no other theoretical energy levels are available in the literature with which to compare, we have performed another calculation from the Flexible Atomic Code (FAC) of Gu (2003), which is easily available from the website http://kipac-tree.standford.edu/fac/. Energy levels obtained from this code are also listed in Table 1, and the excellent agreement between the GRASP and FAC energy levels gives confidence in our results.

\section{Radiative rates}

The absorption oscillator strength $\left(f_{i j}\right)$ and radiative rate $A_{j i}$ (in $\mathrm{s}^{-1}$ ) for a transition $i \rightarrow j$ are related by the following expression:

$f_{i j}=\frac{m c}{8 \pi^{2} e^{2}} \lambda_{j i}{ }^{2} \frac{\omega_{j}}{\omega_{i}} A_{j i}=1.49 \times 10^{-16} \lambda_{j i}^{2}\left(\omega_{j} / \omega_{i}\right) A_{j i}$

where $m$ and $e$ are the electron mass and charge, respectively, $c$ is the velocity of light, $\lambda_{j i}$ is the transition energy/wavelength in $\AA$, and $\omega_{i}$ and $\omega_{j}$ are the statistical weights of the lower $(i)$ and upper $(j)$ levels, respectively. Similarly, the oscillator strength $f_{i j}$ (dimensionless) and the line strength $S$ (in atomic unit, $1 \mathrm{au}=6.460 \times 10^{-36} \mathrm{~cm}^{2} \mathrm{esu}^{2}$ ) are related by the following standard equations:

for the electric dipole (E1) transitions:

$A_{j i}=\frac{2.0261 \times 10^{18}}{\omega_{j} \lambda_{j i}^{3}} S^{\mathrm{E} 1} \quad$ and $\quad f_{i j}=\frac{303.75}{\lambda_{j i} \omega_{i}} S^{\mathrm{E} 1}$, 
Table 1. Energy levels (in Ryd) of Fe XVI.

\begin{tabular}{|c|c|c|c|c|c|c|c|}
\hline Index & Configuration & Level & NIST & GRASP $^{a}$ & GRASP $^{b}$ & $\overline{\text { FAC }^{c}}$ & $\mathrm{SZF}^{d}$ \\
\hline 1 & $3 \mathrm{~s}$ & ${ }^{2} \mathrm{~S}_{1 / 2}$ & 0.0000 & 0.0000 & 0.0000 & 0.0000 & 0.0000 \\
\hline 2 & $3 p$ & ${ }^{2} \mathrm{P}_{1 / 2}^{\circ}$ & 2.5260 & 2.5332 & 2.5346 & 2.5352 & 2.5332 \\
\hline 3 & $3 p$ & ${ }^{2} \mathrm{P}_{3 / 2}^{\circ}$ & 2.7169 & 2.7277 & 2.7246 & 2.7249 & 2.7236 \\
\hline 4 & $3 d$ & ${ }^{2} \mathrm{D}_{3 / 2}$ & 6.1554 & 6.1758 & 6.1680 & 6.1590 & 6.1671 \\
\hline 5 & $3 d$ & ${ }^{2} \mathrm{D}_{5 / 2}$ & 6.1820 & 6.2045 & 6.1936 & 6.1843 & 6.1929 \\
\hline 6 & $4 \mathrm{~s}$ & ${ }^{2} \mathrm{~S}_{1 / 2}$ & 17.0182 & 17.0038 & 16.9944 & 16.9932 & 17.0118 \\
\hline 7 & $4 p$ & ${ }^{2} \mathrm{P}_{1 / 2}^{\circ}$ & 18.0252 & 18.0080 & 17.9995 & 17.9989 & 18.0155 \\
\hline 8 & $4 p$ & ${ }^{2} \mathrm{P}_{3 / 2}^{\circ}$ & 18.0980 & 18.0840 & 18.0736 & 18.0729 & 18.0937 \\
\hline 9 & $4 d$ & ${ }^{2} \mathrm{D}_{3 / 2}$ & 19.3570 & 19.3492 & 19.3371 & 19.3277 & 19.3577 \\
\hline 10 & $4 d$ & ${ }^{2} \mathrm{D}_{5 / 2}$ & 19.3677 & 19.3623 & 19.3490 & 19.3396 & 19.3709 \\
\hline 11 & $4 \mathrm{f}$ & ${ }^{2} \mathrm{~F}_{5 / 2}^{\circ}$ & 19.9077 & 19.8943 & 19.8798 & 19.8865 & 19.9074 \\
\hline 12 & $4 f$ & ${ }^{2} \mathrm{~F}_{7 / 2}^{\circ}$ & 19.9125 & 19.8989 & 19.8842 & 19.8908 & 19.9119 \\
\hline 13 & $5 \mathrm{~s}$ & ${ }^{2} \mathrm{~S}_{1 / 2}$ & 24.2500 & 24.2483 & 24.2361 & 24.2326 & 24.2570 \\
\hline 14 & $5 p$ & ${ }^{2} \mathrm{P}^{\circ}{ }_{1 / 2}$ & 24.7606 & 24.7433 & 24.7316 & 24.7283 & 24.7516 \\
\hline 15 & $5 p$ & ${ }^{2} \mathrm{P}_{3 / 2}^{\circ}$ & 24.7970 & 24.7807 & 24.7681 & 24.7646 & 24.7901 \\
\hline 16 & $5 \mathrm{~d}$ & ${ }^{2} \mathrm{D}_{3 / 2}$ & 25.4065 & 25.3910 & 25.3777 & 25.3709 & 25.4002 \\
\hline 17 & $5 d$ & ${ }^{2} \mathrm{D}_{5 / 2}$ & 25.4116 & 25.3979 & 25.3839 & 25.3771 & 25.4070 \\
\hline 18 & $5 f$ & ${ }^{2} \mathrm{~F}_{5 / 2}^{\circ}$ & 25.6840 & 25.6661 & 25.6515 & 25.6608 & 25.6781 \\
\hline 19 & $5 f$ & ${ }^{2} \mathrm{~F}^{\circ} 7 / 2$ & 25.6870 & 25.6684 & 25.6538 & 25.6630 & 25.6804 \\
\hline 20 & $5 \mathrm{~g}$ & ${ }^{2} \mathrm{G}_{7 / 2}$ & 25.7160 & 25.6924 & 25.6777 & 25.6745 & 25.7090 \\
\hline 21 & $5 \mathrm{~g}$ & ${ }^{2} \mathrm{G}_{9 / 2}$ & 25.7160 & 25.6938 & 25.6791 & 25.6759 & 25.7104 \\
\hline 22 & $6 \mathrm{~s}$ & ${ }^{2} \mathrm{~S}_{1 / 2}$ & 28.0300 & 28.0050 & 27.9917 & 27.9873 & $\ldots \ldots$ \\
\hline 23 & $6 p$ & ${ }^{2} \mathrm{P}^{\circ}{ }_{1 / 2}$ & 28.3070 & 28.2843 & 28.2713 & 28.2673 & ….... \\
\hline 24 & $6 p$ & ${ }^{2} \mathrm{P}_{3 / 2}^{\circ}$ & 28.3300 & 28.3055 & 28.2920 & 28.2883 & $\ldots \ldots$. \\
\hline 25 & $6 \mathrm{~d}$ & ${ }^{2} \mathrm{D}_{3 / 2}$ & 28.6691 & 28.6473 & 28.6333 & 28.6290 & ….... \\
\hline 26 & $6 \mathrm{~d}$ & ${ }^{2} \mathrm{D}_{5 / 2}$ & 28.6745 & 28.6513 & 28.6370 & 28.6330 & ........ \\
\hline 27 & $6 f$ & ${ }^{2} \mathrm{~F}^{\circ}{ }_{5 / 2}$ & 28.8245 & 28.8049 & 28.7902 & 28.7936 & ….... \\
\hline 28 & $6 f$ & ${ }^{2} \mathrm{~F}^{\circ}{ }_{7 / 2}$ & 28.8251 & 28.8062 & 28.7915 & 28.7949 & $\ldots \ldots$. \\
\hline 29 & $6 \mathrm{~g}$ & ${ }^{2} \mathrm{G}_{7 / 2}$ & ........ & 28.8220 & 28.8073 & 28.8064 & ........ \\
\hline 30 & $6 \mathrm{~g}$ & ${ }^{2} \mathrm{G}_{9 / 2}$ & ........ & 28.8229 & 28.8081 & 28.8072 & ....... \\
\hline 31 & $7 \mathrm{~s}$ & ${ }^{2} \mathrm{~S}_{1 / 2}$ & 30.2800 & 30.2027 & 30.1888 & 30.1860 & ........ \\
\hline 32 & $7 p$ & ${ }^{2} \mathrm{P}_{1 / 2}^{\circ}$ & ......... & 30.3753 & 30.3617 & 30.3595 & ….... \\
\hline 33 & $7 p$ & ${ }^{2} \mathrm{P}_{3 / 2}^{\circ}$ & 30.4400 & 30.3884 & 30.3744 & 30.3724 & $\ldots \ldots$. \\
\hline 34 & $7 d$ & ${ }^{2} \mathrm{D}_{3 / 2}$ & 30.6230 & 30.5993 & 30.5850 & 30.5826 & ….... \\
\hline 35 & $7 d$ & ${ }^{2} \mathrm{D}_{5 / 2}$ & 30.6250 & 30.6018 & 30.5873 & 30.5850 & ........ \\
\hline 36 & $7 f$ & ${ }^{2} \mathrm{~F}_{5 / 2}^{\circ}$ & 30.7194 & 30.6978 & 30.6832 & 30.6828 & $\cdots$ \\
\hline 37 & $7 \mathrm{f}$ & ${ }^{2} \mathrm{~F}^{\circ} 7 / 2$ & 30.7207 & 30.6987 & 30.6840 & 30.6836 & ........ \\
\hline 38 & $7 \mathrm{~g}$ & ${ }^{2} \mathrm{G}_{7 / 2}$ & ........ & 30.7093 & 30.6946 & 30.6949 & ....... \\
\hline 39 & $7 \mathrm{~g}$ & ${ }^{2} \mathrm{G}_{9 / 2}$ & ........ & 30.7099 & 30.6951 & 30.6954 & $\ldots \ldots$. \\
\hline
\end{tabular}

NIST: http://physics.nist.gov/PhysRefData

${ }^{a}$ Coulomb energies.

${ }^{b}$ QED corrected energies.

${ }^{c}$ Energies from the Flexible Atomic Code of Gu (2003).

${ }^{d}$ Sampson et al. (1990).

for the magnetic dipole (M1) transitions:

$A_{j i}=\frac{2.6974 \times 10^{13}}{\omega_{j} \lambda_{j i}^{3}} S^{\mathrm{M} 1}$ and $\quad f_{i j}=\frac{4.044 \times 10^{-3}}{\lambda_{j i} \omega_{i}} S^{\mathrm{M} 1}$,

for the electric quadrupole (E2) transitions:

$A_{j i}=\frac{1.1199 \times 10^{18}}{\omega_{j} \lambda_{j i}^{5}} S^{\mathrm{E} 2} \quad$ and $\quad f_{i j}=\frac{167.89}{\lambda_{j i}^{3} \omega_{i}} S^{\mathrm{E} 2}$, and for the magnetic quadrupole (M2) transitions:

$A_{j i}=\frac{1.4910 \times 10^{13}}{\omega_{j} \lambda_{j i}^{5}} S^{\mathrm{M} 2} \quad$ and $\quad f_{i j}=\frac{2.236 \times 10^{-3}}{\lambda_{j i}^{3} \omega_{i}} S^{\mathrm{M} 2}$.

In Table 2 we present transition energies $\left(\Delta E_{i j}\right.$ in $\AA$ ), radiative rates $\left(A_{j i}\right.$ in $\left.\mathrm{s}^{-1}\right)$, oscillator strengths $\left(f_{i j}\right.$, dimensionless), and line strengths ( $S$ in au), in length form only, for all

(4) 221 electric dipole (E1) and 278 electric quadrupole (E2) transitions among the 39 levels of Fe XVI. The indices used to 
represent the lower and upper levels of a transition have already been defined in Table 1 . Similar results for 203 magnetic dipole (M1) and 231 magnetic quadrupole (M2) transitions are listed in Table 3 . These results not only cover a wider range of transitions among a larger number of levels than hitherto available in the literature, but are also for all possible transitions among the above four types.

The other similar results available in the literature with which to compare are those of Cornille et al. (1997) and Eissner et al. (1999) for E1 transitions. All three sets of $A$-values agree within $10 \%$ for the common transitions, including the 4-8 $\left(3 \mathrm{~d}^{2} \mathrm{D}_{3 / 2}-4 \mathrm{p}^{2} \mathrm{P}_{3 / 2}^{\circ}\right)$ transition for which the earlier reported $f$-value by Tayal (1994) is lower by an order of magnitude - see Table 3 of Eissner et al. It may also be noted that Eissner et al. have listed the $f$-value of Cornille et al. as 0.642 which (perhaps) follows from a misprint in their Table 2 (i.e. $2.566 \mathrm{E}-2$ instead of $2.566 \mathrm{E} 0$ for $\omega_{i} f_{i j}$ ), because their listed $A$ value for this transition is $7.313 \times 10^{9} \mathrm{~s}^{-1}$, which agrees very well with our present result of $7.366 \times 10^{9} \mathrm{~s}^{-1}$. Therefore, the $f$-value of Cornille et al. for the 4-8 transition should be 0.00642 , which is in complete agreement with ours and the calculations of Eissner et al. Similarly, a satisfactory agreement among the $\Omega$ values of Tayal, Cornille et al. and our present calculations for this transition suggests that there is a misprint in the Tayal listing in his Table 3 , and his $f$-value is actually 0.0063 in stead of 0.063 . However, this comparison of $A$-values is so far confined to transitions among the lowest 19 levels only. Therefore, we have performed another independent calculation from FAC with $n \leq 7$ configurations. A comparison made between the $A$-values from GRASP and FAC indicates excellent agreement within $10 \%$, except for 5 transitions (namely 2-31, 3-31, 11-34, 11-35 and 12-35). However, all of these five transitions are comparatively weak $(f \leq 0.001)$, and the differences are below $30 \%$. Therefore, we may state with confidence that for transitions in Fe XVI there is no discrepancy among various theoretical calculations for the radiative rates, and the results listed in Tables 2 and 3 are probably accurate to better than $10 \%$.

\section{Collision strengths}

For the computations of collision strengths, we have employed the DARC program of Norrington \& Grant (2006). This program includes the relativistic effects in a systematic way, in both the target description and the scattering model. It is based on the $j j$ coupling scheme, and uses the Dirac-Coulomb Hamiltonian in the $R$-matrix approach. However, because of the inclusion of fine-structure in the definition of channel coupling, the matrix size of the Hamiltonian increases substantially. The $R$-matrix radius has been adopted to be 9.84 au, and 62 continuum orbitals have been included for each channel angular momentum for the expansion of the wavefunction. This allows us to compute values of $\Omega$ up to an energy of 220 Ryd. The maximum number of channels for a partial wave is 200 , and the corresponding size of the Hamiltonian matrix is 12553 . In order to obtain convergence of $\Omega$ for all transitions and at all energies, we have included all partial waves with angular momentum $J \leq 39$, although a higher range would have been

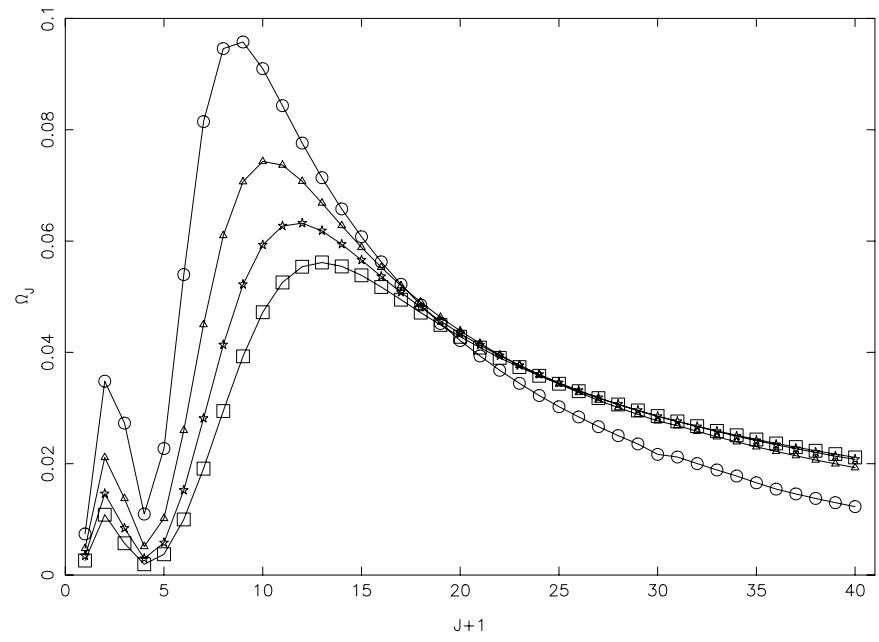

Fig. 1. Partial collision strengths for the $3 \mathrm{~s}^{2} \mathrm{~S}_{1 / 2}-3 \mathrm{p}^{2} \mathrm{P}^{\circ}{ }_{1 / 2}(1-2)$ transition of Fe XVI, at three energies of: 50 Ryd (circles), 100 Ryd (triangles), 150 Ryd (stars), and 200 Ryd (squares).

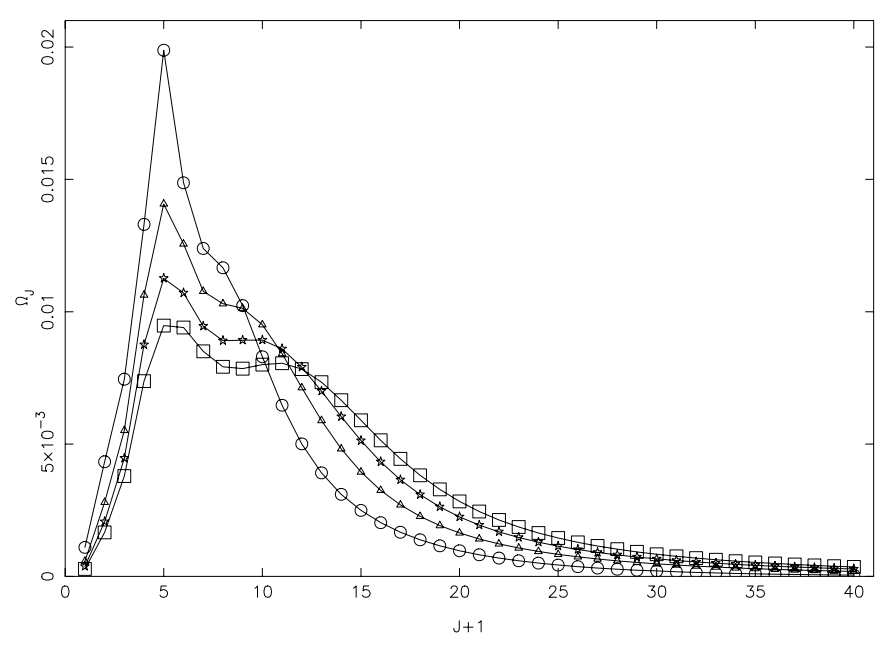

Fig. 2. Partial collision strengths for the $3 \mathrm{~s}^{2} S_{1 / 2}-3 d{ }^{2} D_{3 / 2}(1-4)$ transition of Fe XVI, at three energies of: 50 Ryd (circles), 100 Ryd (triangles), 150 Ryd (stars), and 200 Ryd (squares).

preferable for the convergence of allowed transitions. However, to account for the inclusion of higher neglected partial waves, we have included a top-up, based on the Coulomb-Bethe approximation for allowed transitions and geometric series for forbidden ones.

In Figs. $1-3$ we show the variation of $\Omega$ with angular momentum $J$ at four energies of 50, 100, 150 and 200 Ryd, and for three transitions, namely $1-2\left(3 \mathrm{~s}^{2} \mathrm{~S}_{1 / 2}-3 \mathrm{p}{ }^{2} \mathrm{P}^{\circ}{ }_{1 / 2}\right), 1-4$ $\left(3 \mathrm{~s}^{2} \mathrm{~S}_{1 / 2}-3 \mathrm{~d}{ }^{2} \mathrm{D}_{3 / 2}\right)$ and $2-3\left(3 \mathrm{p}^{2} \mathrm{P}^{\circ}{ }_{1 / 2}-3 \mathrm{p}^{2} \mathrm{P}^{\circ}{ }_{3 / 2}\right)$. It is clear from Fig. 1 that the range of partial waves included in the present work is insufficient for the convergence of $\Omega$ for the allowed transitions. However, it is fully sufficient for the convergence of forbidden transitions, as shown in Figs. 2 and 3. These two figures also demonstrate that the partial waves ranges adopted by Tayal (1994) and Cornille et al. (1997), i.e. $J \leq 15$ and $L \leq 25$, respectively, are insufficient to achieve convergence in $\Omega$ for a majority of transitions. This is the main reason 


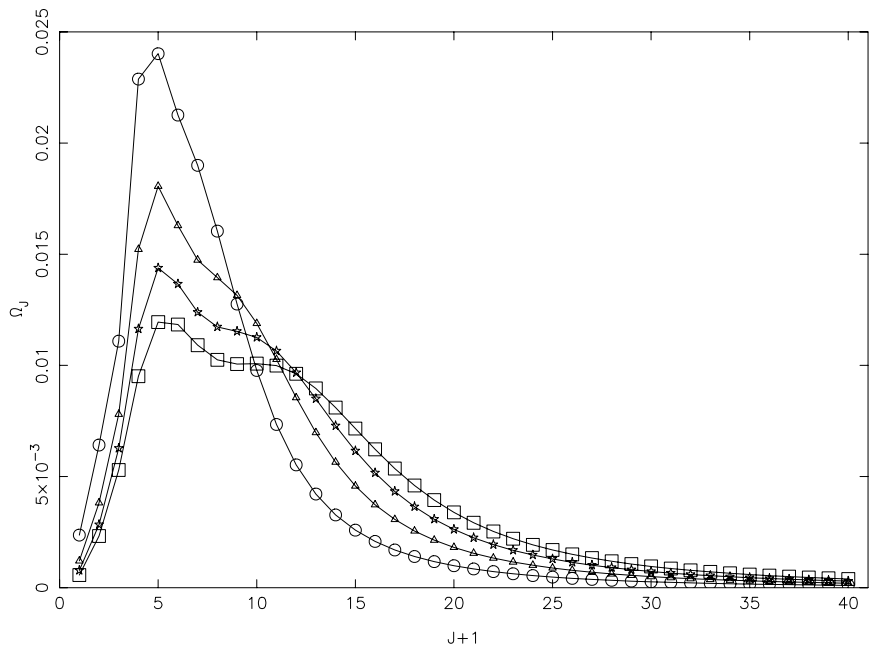

Fig. 3. Partial collision strengths for the $3 \mathrm{p}^{2} \mathrm{P}^{\circ}{ }_{1 / 2}-3 \mathrm{p}^{2} \mathrm{P}_{3 / 2}^{\circ}(2-3)$ transition of Fe XVI, at three energies of: 50 Ryd (circles), 100 Ryd (triangles), 150 Ryd (stars), and 200 Ryd (squares).

for the large differences observed by Eissner et al. (1999) with the $\Omega$ values of Tayal and Cornille et al. In Table 4 we present our results of $\Omega$ for transitions from the lowest 5 levels to higher excited levels, at four energies of 50, 100, 150 and 200 Ryd.

Since Eissner et al. (1999) have not reported any $\Omega$ values, no comparisons can be made with their results. However, we have made comparisons with the DW data of Sampson et al. (1990), which are stored in the CHIANTI database in the form of analytical expressions. Unfortunately, these results for $\Omega$ are confined to transitions from the lowest 5 levels to the higher excited levels up to 21 . Nevertheless, a comparison made between the present DARC and earlier DW values of $\Omega$ over a wide energy range of $50 \leq E \leq 200$ Ryd, shows excellent agreement within $10 \%$ for all transitions. This is highly encouraging and confirms the accuracy of our results. However, a similar comparison made with the DW values of $\Omega$ from Cornille et al. (1997) shows large discrepancies (up to a factor of four) for many transitions, such as: $6-16\left(4 \mathrm{~s}^{2} \mathrm{~S}_{1 / 2}-5 \mathrm{~d}{ }^{2} \mathrm{D}_{3 / 2}\right), 7-15$ $\left(4 \mathrm{p}^{2} \mathrm{P}^{\circ}{ }_{1 / 2}-5 \mathrm{p}^{2} \mathrm{P}_{3 / 2}^{\circ}\right)$, and $8-14\left(4 \mathrm{p}^{2} \mathrm{P}_{3 / 2}^{\circ}-5 \mathrm{p}^{2} \mathrm{P}_{1 / 2}^{\circ}\right)$. All of these are forbidden transitions and the $\Omega$ values of Cornille et al. are invariably smaller. As an example, we demonstrate in Fig. 4 the $\Omega$ values of ours and Cornille et al. for three transitions, namely 6-16 (4s $\left.{ }^{2} \mathrm{~S}_{1 / 2}-5 \mathrm{~d}{ }^{2} \mathrm{D}_{3 / 2}\right), 6-17\left(4 \mathrm{~s}{ }^{2} \mathrm{~S}_{1 / 2}-5 \mathrm{~d}\right.$ $\left.{ }^{2} \mathrm{D}_{5 / 2}\right)$ and 7-15 (4p $\left.{ }^{2} \mathrm{P}_{1 / 2}^{\circ}-5 \mathrm{p}^{2} \mathrm{P}_{3 / 2}^{\circ}\right)$. Clearly, differences between the two sets of $\Omega$ increase with increasing energy, and are due to the inclusion of a limited range of partial waves by Cornille et al., as discussed above and also highlighted by Eissner et al.

For transitions involving the higher excited levels of the $n=6$ and 7 configurations, no comparisons of $\Omega$ values are possible, because of the paucity of data in the literature. Therefore, as for the $A$-values, we have performed a calculation with FAC, which is based on the DW method and also includes relativistic effects. As expected, the two sets of $\Omega$ values generally agree within $20 \%$ for a majority of transitions. We will further discuss the $\Omega$ values from FAC in the following section.

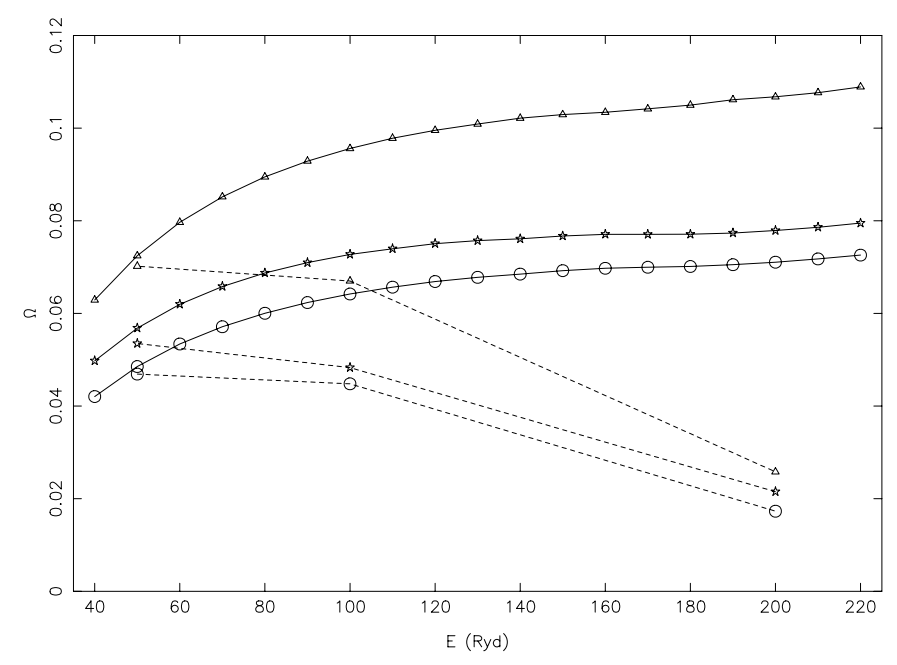

Fig. 4. Comparison of collision strengths for some transitions of Fe XVI. Continuous curve: present results, broken curve: Cornille et al. (1997), circles: 6-16, triangles: 6-17, and stars: 7-15 transition.

\section{Effective collision strengths}

Effective collision strengths $\Upsilon$ are obtained after integrating $\Omega$ over a Maxwellian distribution of electron velocities, i.e.

$\Upsilon\left(T_{\mathrm{e}}\right)=\int_{0}^{\infty} \Omega(E) \exp \left(-E_{j} / k T_{\mathrm{e}}\right) \mathrm{d}\left(E_{j} / k T_{\mathrm{e}}\right)$

where $E_{j}$ is the incident energy of the electron with respect to the final state of the transition, $k$ is Boltzmann's constant, and $T_{\mathrm{e}}$ is the electron temperature in K. Once the value of $\Upsilon$ is known for a transition, the corresponding value of the excitation $q(i, j)$ and de-excitation $q(j, i)$ rate coefficients can be easily obtained from the following simple relations:

$q(i, j)=\frac{8.63 \times 10^{-6}}{\omega_{i} T_{\mathrm{e}}^{1 / 2}} \Upsilon \exp \left(-E_{i j} / k T_{\mathrm{e}}\right) \quad \mathrm{cm}^{3} \mathrm{~s}^{-1}$

and

$q(j, i)=\frac{8.63 \times 10^{-6}}{\omega_{j} T_{\mathrm{e}}^{1 / 2}} \Upsilon \quad \mathrm{cm}^{3} \mathrm{~s}^{-1}$,

where $\omega_{i}$ and $\omega_{j}$ are the statistical weights of the initial $(i)$ and final $(j)$ states, respectively, and $E_{i j}$ is the transition energy.

Since the threshold energy region is dominated by numerous resonances, values of $\Omega$ have been computed at a large number of energies in order to delineate these resonances. We have performed our calculations of $\Omega$ at $\sim 15400$ energies in the threshold region. Close to thresholds ( $\sim 0.1$ Ryd above a threshold) the energy mesh is $0.001 \mathrm{Ryd}$, and away from thresholds is 0.002 Ryd. Thus care has been taken to include as many resonances as possible, and with as fine a resolution as is computationally feasible. The density and importance of resonances can be appreciated from Figs. 5-7, in which we show our values of $\Omega$ in the thresholds region for the $1-2\left(3 \mathrm{~s}^{2} \mathrm{~S}_{1 / 2}-3 \mathrm{p}{ }^{2} \mathrm{P}^{\circ}{ }_{1 / 2}\right)$, $1-3\left(1 \mathrm{~s}^{2} \mathrm{~S}_{1 / 2}-3 \mathrm{p}^{2} \mathrm{P}_{3 / 2}^{\circ}\right)$ and $2-3\left(3 \mathrm{p}^{2} \mathrm{P}_{1 / 2}^{\circ}-3 \mathrm{p}^{2} \mathrm{P}_{3 / 2}^{\circ}\right)$ transitions, respectively. Resonances for these transitions are particularly dense at energies below $\sim 6 \mathrm{Ryd}$, and hence contribute to the values of $\Upsilon$ mainly at lower temperatures. Tayal (1994) 


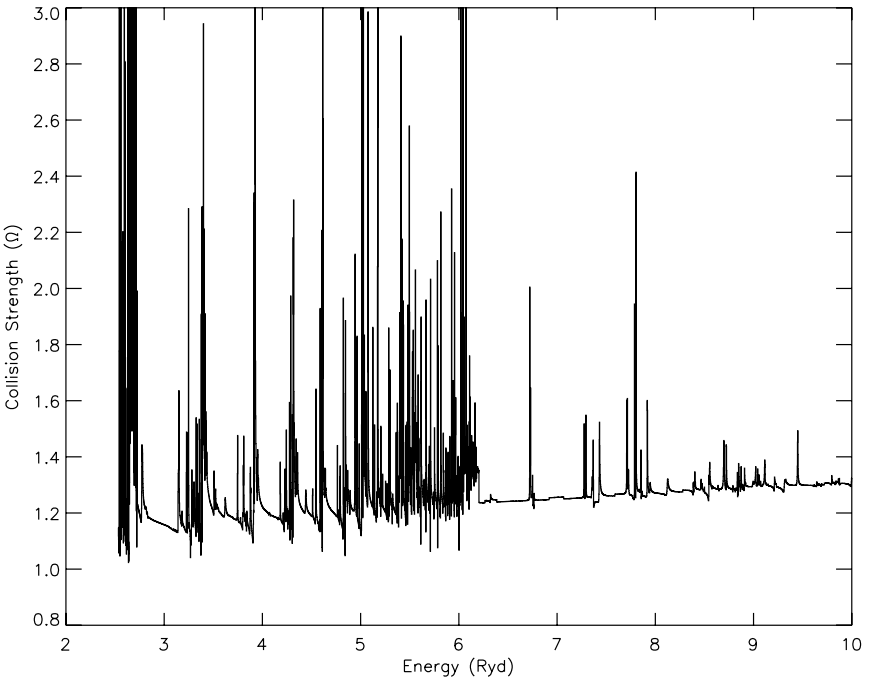

Fig. 5. Collision strengths for the $3 s^{2} S_{1 / 2}-3 p{ }^{2} \mathrm{P}^{\circ}{ }_{1 / 2}(1-2)$ transition of Fe XVI.

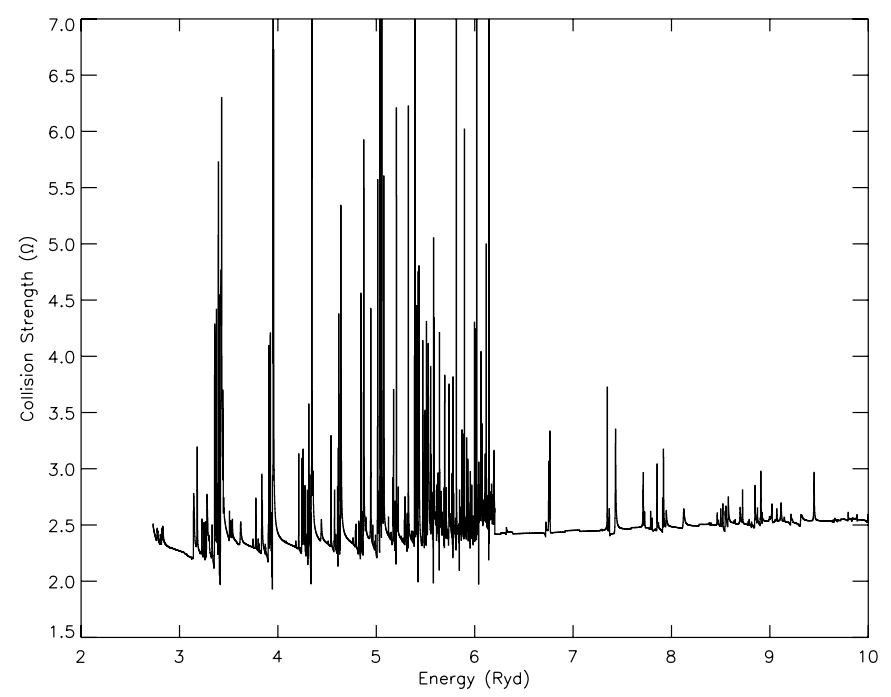

Fig. 6. Collision strengths for the $3 s^{2} \mathrm{~S}_{1 / 2}-3 p{ }^{2} \mathrm{P}_{3 / 2}^{\circ}(1-3)$ transition of Fe XVI.

has shown similar resonances for the 1-2 and 1-3 transitions, which compare well with our results in Figs. 1 and 2, except that our resonances are denser because we have adopted a finer mesh of electron energy.

Our calculated values of $\Upsilon$ are listed in Table 5 over a wide temperature range of $10^{5}-10^{7} \mathrm{~K}$, which is fully sufficient for applications in solar and astrophysical plasmas. Before we compare our values for $\Upsilon$, we would like to note that the energy range of our calculations, i.e. $220 \mathrm{Ryd}$, is nearly sufficient to obtain converged results for all transitions up to $T_{\mathrm{e}}=10^{7} \mathrm{~K}$. However, a wider energy range would be preferable particularly for the higher end of the temperature range. Therefore, taking advantage of a better than $20 \%$ agreement between our DARC and FAC values of $\Omega$, we have supplemented our DARC data in the $220 \leq E \leq 300$ Ryd energy range from FAC in order to achieve better convergence of $\Upsilon$ values at all temperatures. This widening of the energy range has affected only a few transitions, and the changes are less than $10 \%$ at the highest

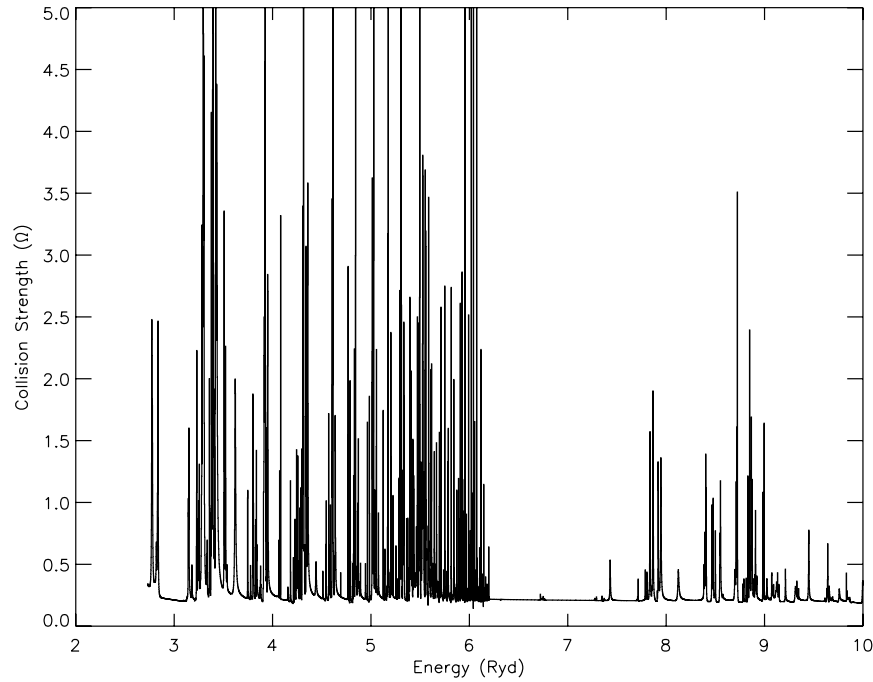

Fig. 7. Collision strengths for the $3 \mathrm{p}^{2} \mathrm{P}^{\circ}{ }_{1 / 2}-3 \mathrm{p}^{2} \mathrm{P}_{3 / 2}^{\circ}(2-3)$ transition of Fe XVI

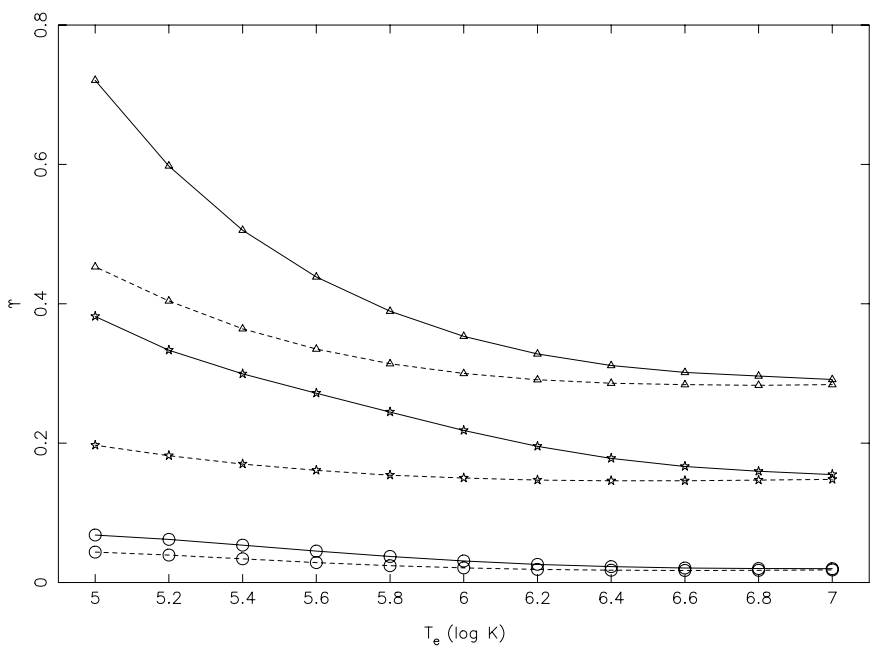

Fig. 8. Comparison of effective collision strengths for some transitions of Fe XVI. Continuous curve: present results, broken curve: Eissner et al. (1999), circles: $2-8$, triangles: $4-9$, and stars: 7-10 transition.

temperature of our calculations, i.e. $10^{7} \mathrm{~K}$. Therefore, we can state with confidence that the integral in Eq. (6) has converged, and a further widening of energy range will be practically ineffective.

Since the $\Upsilon$ values of Eissner et al. (1999) are comparatively more accurate than those of Tayal (1994), we will only make comparisons of our present results with their values. Differences up to $50 \%$ between the two sets of $\Upsilon$ are frequent for many common transitions, such as $2-8,4-9$ and 7-10, as shown in Fig. 8. However, for all of these (and many more) transitions, differences in the two sets of $\Upsilon$ are the highest at the lowest temperature, and continuously decrease with increasing temperature. Nevertheless, considering that Eissner et al. have adopted the $R$-matrix approach, have included CI in the construction of wavefunctions, have included relativistic effects in calculating values of $\Omega$, have resolved resonances in the thresholds region with a comparably fine energy mesh, and have taken care to ensure the convergence of $\Omega$ as well as of $\Upsilon$, 


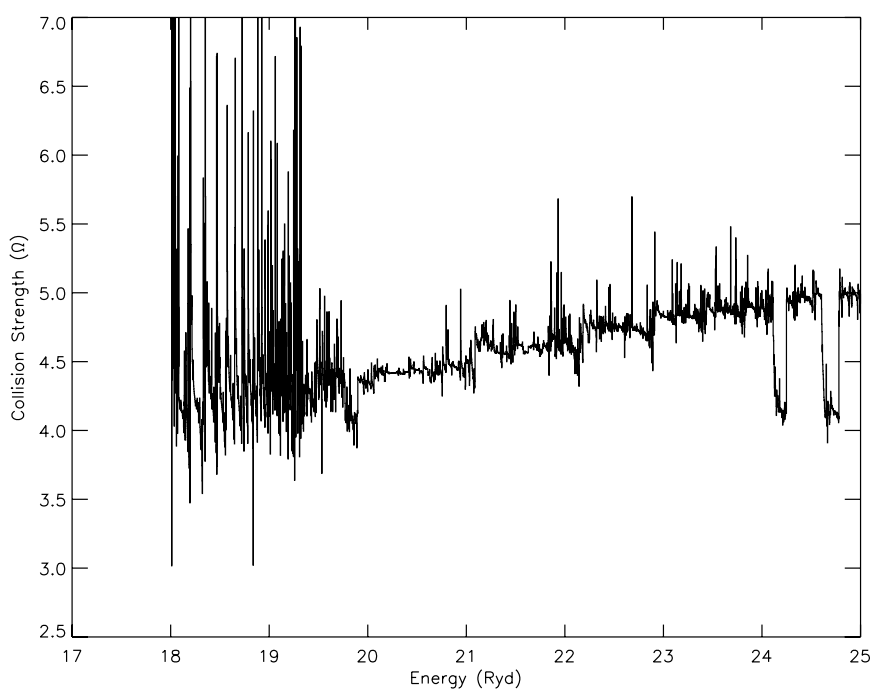

Fig. 9. Collision strengths for the $4 \mathrm{~s}^{2} \mathrm{~S}_{1 / 2}-4 \mathrm{p}{ }^{2} \mathrm{P}^{\circ}{ }_{1 / 2}(6-7)$ transition of Fe XVI.

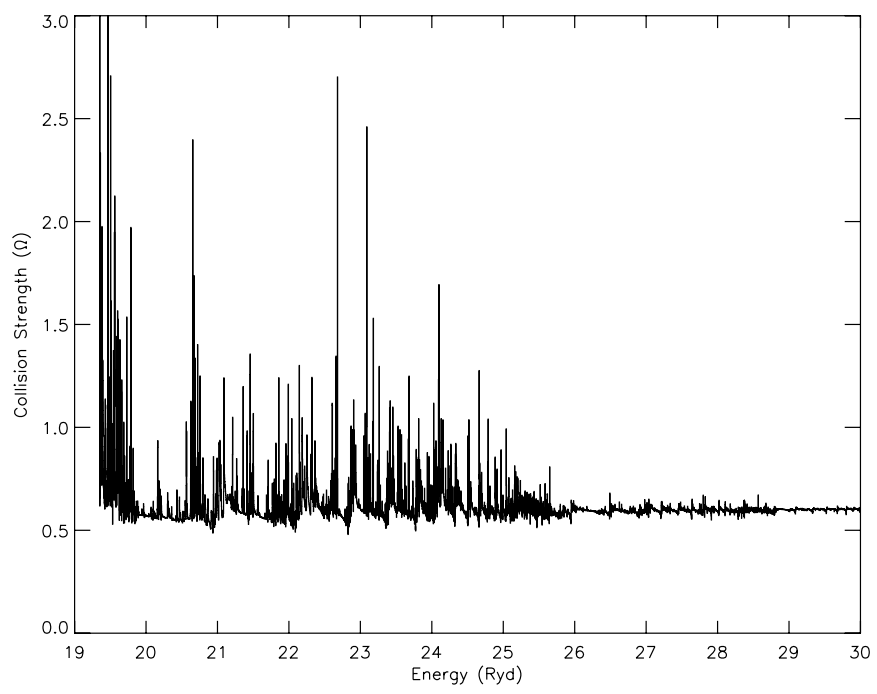

Fig. 10. Collision strengths for the $4 \mathrm{~s}^{2} S_{1 / 2}-4 d{ }^{2} D_{3 / 2}$ (6-9) transition of Fe XVI

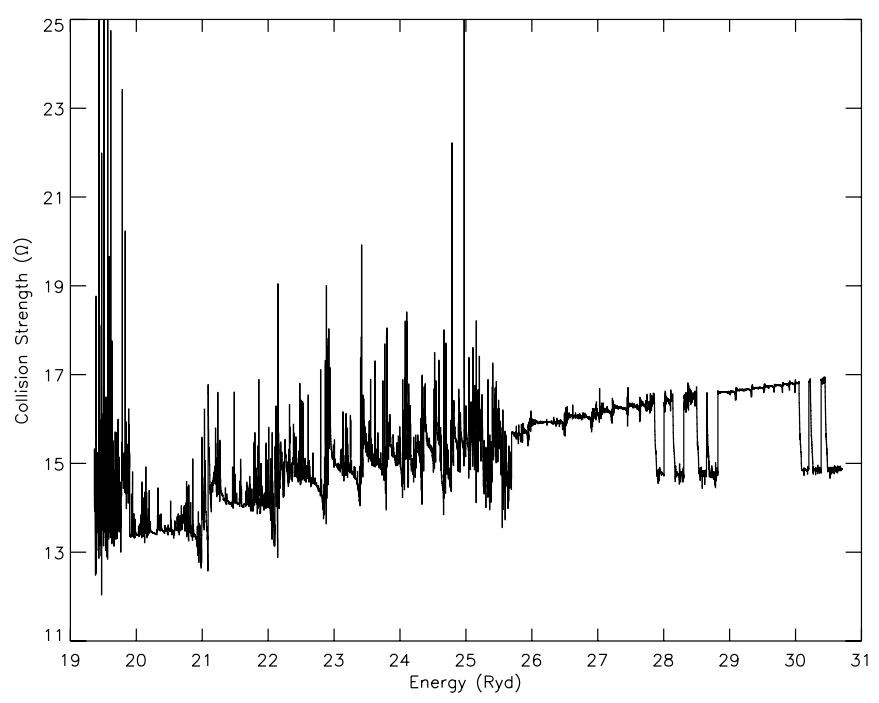

Fig. 11. Collision strengths for the $4 \mathrm{p}^{2} \mathrm{P}_{3 / 2}^{\circ}-4 \mathrm{~d}^{2} \mathrm{D}_{5 / 2}(8-10)$ transition of Fe XVI. such large differences between the two calculations are unexpected, and therefore, need to be elaborated and understood. We discuss these below in detail.

Since Eissner et al. (1999) have shown resonances for the $6-7\left(4 \mathrm{~s}^{2} \mathrm{~S}_{1 / 2}-4 \mathrm{p}^{2} \mathrm{P}^{\circ}{ }_{1 / 2}\right), 6-9\left(4 \mathrm{~s}^{2} \mathrm{~S}_{1 / 2}-4 \mathrm{~d}^{2} \mathrm{D}_{3 / 2}\right)$, and 8-10 (4p $\left.{ }^{2} \mathrm{P}_{3 / 2}^{\circ}-4 \mathrm{~d}^{2} \mathrm{D}_{5 / 2}\right)$ transitions, we show in Figs. 9-11 our values of $\Omega$ for the same three transitions, which will facilitate a direct comparison between the two calculations, and hence the differences in values of $\Upsilon$. As is clear from Fig. 9, resonances for the 6-7 transition dominate in a small energy region below 20 Ryd, which is also shown in Fig. 5 of Eissner et al. However, the variation of $\Omega$ is different in the two calculations, as in Eissner et al. work the background $\Omega$ suddenly rises and then considerably drops, whereas in our case it continuously rises, as it is an allowed transition. The sudden rise of background $\Omega$ in the low energy region in Fig. 5 of Eissner et al. is (perhaps) a result of some numerical problem in the Breit-Pauli $R$-matrix code they adopted. Similarly, at energies above thresholds the behaviour of their $\Omega$ values is anomalous for some transitions, such as in Figs. 4 and 5, which they themselves have discussed. To avoid this problem they have suggested the inclusion of a larger range of partial waves (i.e. $J>40$ ). However, our calculations within the same range of partial waves do not show such anomalous behaviour. Anyway, as a result of these differences our values of $\Upsilon$ are higher for the 6-7 transition by up to $40 \%$, particularly at lower temperatures. As the temperature increases, the discrepancy between the two sets of $\Upsilon$ decreases, and completely disappears at $T_{\mathrm{e}}=10^{7} \mathrm{~K}$, because both calculations have ensured convergence of $\Omega$ at higher energies.

For the 6-9 transition shown in Fig. 10, two distinct differences are noted with the corresponding Fig. 6 of Eissner et al. (1999). Firstly, close to the threshold we observe denser and higher resonances, which are due to the adoption of the $j j$ coupling scheme in our calculations. Secondly, and more importantly, resonances in our work are spread over a wider energy range of $\sim 6$ Ryd before converging on to the higher thresholds. This is because we have now included an additional 27 levels in our calculations. Therefore, as expected, for this transition our values of $\Upsilon$ are also higher by up to $20 \%$, but at lower temperatures only. Finally, our Fig. 11 for the 8-10 transition does not show any anomalous behaviour in values of $\Omega$, as seen in the corresponding Fig. 4 of Eissner et al. Similarly, for this transition also resonances are spread over a wider energy range. As a combined effect of both of these differences, the two sets of $\Upsilon$ values differ up to $40 \%$, but differences continuously decrease with increasing temperature.

Before discussing further comparisons among the $\Upsilon$ values, we would like to comment on some anomalies observed in our Figs. 9 and 11, which show sudden dips in background $\Omega$, particularly at energies above 27 Ryd. Similar dips have also been observed in calculations for other ions, such as Fe XV, as shown in Fig. 3a of Aggarwal et al. (2001), and arise because of the numerical difficulties in the stage DSTGF of the program when it automatically switches IPERT (the perturbation parameter which accounts for the long-range potentials) from 1 to 0 . This results in underestimation of $\Omega$ values up to $\sim 25 \%$. However, it happens only for a few transitions and in narrow energy ranges, as seen in Figs. 9 and 11, and does not 


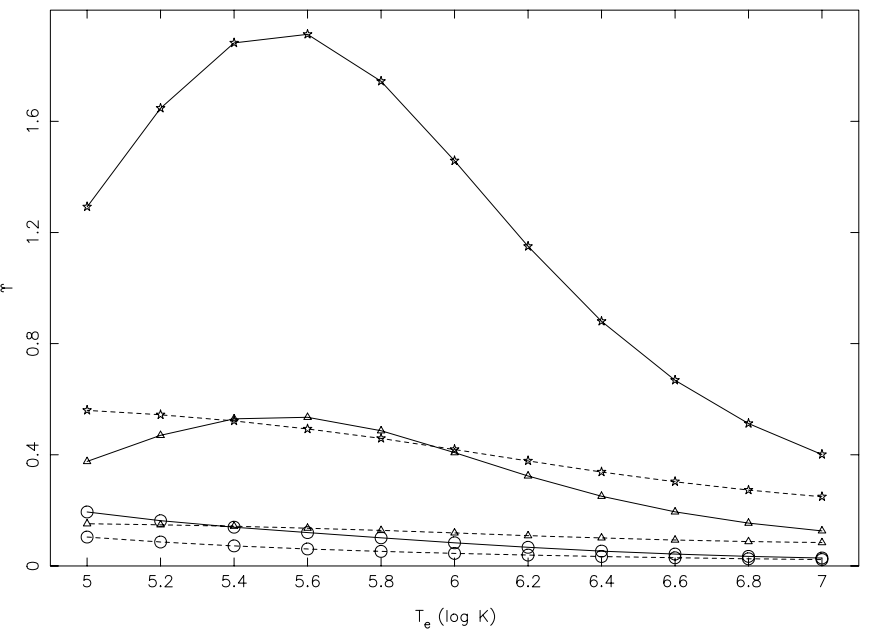

Fig. 12. Comparison of effective collision strengths for some transitions of Fe XVI. Continuous curve: present results, broken curve: Eissner et al. (1999), circles: 4-10, triangles: 9-12, and stars: 11-12 transition.

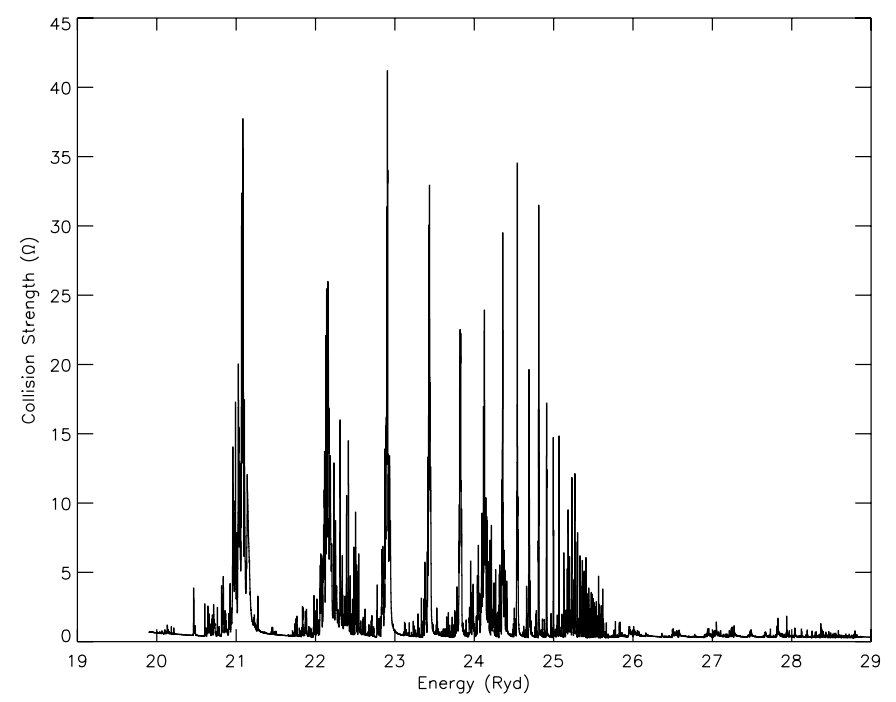

Fig. 13. Collision strengths for the $4 \mathrm{f}^{2} \mathrm{~F}_{5 / 2}^{\circ}-4 \mathrm{f}{ }^{2} \mathrm{~F}_{7 / 2}^{\circ}$ (11-12) transition of Fe XVI.

affect the overall accuracy of the $\Upsilon$ values. For example, an exercise performed for the $8-10$ transition by removing the dips, yielded results for $\Upsilon$ within $1 \%$ of those listed in Table 5. This is much less than the accuracy estimates of our calculations.

For the transitions discussed above, differences in our present and earlier (Eissner et al. 1999) values of $\Upsilon$ are below $50 \%$. However, there are some forbidden transitions, namely 4-10, 5-9, 7-12, 9-10, 9-12 and 11-12, for which the two sets of $\Upsilon$ differ by up to a factor of three as shown in Fig. 12 for the $4-10\left(3 d^{2} D_{3 / 2}-4 d^{2} D_{5 / 2}\right), 9-12\left(4 d^{2} D_{3 / 2}-4 f\right.$ $\left.{ }^{2} \mathrm{~F}_{7 / 2}^{\circ}\right)$ and $11-12\left(4 \mathrm{f}^{2} \mathrm{~F}^{\circ}{ }_{5 / 2}-4 \mathrm{f}^{2} \mathrm{~F}^{\circ}{ }_{7 / 2}\right)$ transitions. For all of these (and other) transitions, differences in the two sets of $\Upsilon$ are dominant at the lower temperatures, which are clearly due to the inclusion of resonances from the higher excited levels. As an example, we demonstrate in Fig. 13 our resonances for the 11-12 transition. Since these resonances are spread over a wide energy range of $\sim 6$ Ryd (equivalent to $\sim 10^{6} \mathrm{~K}$ ), their contribution has enhanced the values of $\Upsilon$ up to a factor of three, depending on the temperature. Therefore, the present work is a clear improvement over that of Eissner et al. However, we would like to warn that our listed results of $\Upsilon$ may similarly be underestimated, especially for transitions involving the levels of the $n \geq 6$ configurations, and a larger calculation than presently performed may be necessary in order to improve upon the accuracy of $\Upsilon$ values for such transitions.

\section{Conclusions}

In the present work, results for energy levels, radiative rates, collision strengths, and effective collision strengths for transitions among the lowest 39 levels of Fe XVI have been presented for all transitions. Additionally, results for radiative rates have been presented for four types of transitions, namely E1, E2, M1 and M2. A complete set of results are likely to be useful for the modelling of a variety of plasmas.

Additionally, our calculations have been performed in the $j j$ coupling scheme, CI and relativistic effects have been included while generating wavefunctions, and a large range of partial waves has been adopted in order to achieve convergence in the values of $\Omega$ for a majority of the transitions. Furthermore, resonances have been resolved in a fine energy mesh in order to improve upon the accuracy of the derived values of $\Upsilon$. Similarly, values of $\Omega$ have been computed over a wider energy range (up to $300 \mathrm{Ryd}$ ) in order to determine values of $\Upsilon$ up to a temperature of $10^{7} \mathrm{~K}$. Based on comparisons made with earlier available data for several atomic parameters, our energy levels are assessed to be accurate to $\sim 0.1 \%$, whereas the accuracy of the radiative rates is probably better than $10 \%$. However, because of the presence of numerous resonances in collision strengths in the thresholds region, values of $\Upsilon$ may only be accurate to $\sim 20 \%$, particularly towards the lower end of the temperature range.

A comparison of our results for effective collision strengths with the corresponding data of Eissner et al. (1999) indicates that their values of $\Upsilon$ for some transitions are underestimated by up to a factor of three, particularly towards the lower end of the temperature range of $10^{5}-10^{7} \mathrm{~K}$. This is because they performed calculations among the lowest 12 levels (66 transitions) only, whereas we have extended that range up to 39 levels (741 transitions). Inclusion of a larger range of levels, and hence of transitions, has enabled us to take account of the resonances arising from the higher excited levels, which has helped in improving upon the accuracy of $\Upsilon$ values, particularly for transitions among the levels of the $n \leq 5$ configurations.

Acknowledgements. This work has been financed by the Engineering and Physical Sciences and Particle Physics and Astronomy Research Councils of the UK. We would like to thank Dr. Patrick Norrington for providing his DARC code prior to publication, and F.P.K. is grateful to A.W.E. Aldermaston for the award of a William Penny Fellowship. Finally, we thank an anonymous referee for his thoughtful and constructive comments. 


\section{References}

Acton, L. W., Bruner, M. E., Brown, W. A., et al. 1985, ApJ, 291, 865 Aggarwal, K. M., \& Keenan, F. P. 1999, J. Phys. B, 32, 3585 Aggarwal, K. M., \& Keenan, F. P. 2001, Phys. Scr., 64, 439 Aggarwal, K. M., \& Keenan, F. P. 2003a, MNRAS, 338, 412 Aggarwal, K. M., \& Keenan, F. P. 2003b, A\&A, 399, 799 Aggarwal, K. M., \& Keenan, F. P. 2004a, A\&A, 418, 371 Aggarwal, K. M., \& Keenan, F. P. 2004b, A\&A, 427, 763 Aggarwal, K. M., \& Keenan, F. P. 2005a, A\&A, 429, 1117 Aggarwal, K. M., \& Keenan, F. P. 2005b, A\&A, 439, 1215

Aggarwal, K. M., Keenan, F. P., \& Kisielius, R. 2004, A\&A, 420, 783 Aggarwal, K. M., Keenan, F. P., \& Msezane, A. Z. 2001, J. Phys. B, 34, L757

Aggarwal, K. M., Keenan, F. P., \& Msezane, A. Z. 2003a, A\&A, 410, 349

Aggarwal, K. M., Keenan, F. P., \& Msezane, A. Z. 2003b, ApJS, 144, 169

Behring, W. E., Cohen, L., Feldman, U., \& Doschek, G. A. 1976, ApJ, 203, 521

Berrington, K. A., Burke, P. G., LeDourneuf, M., et al. 1978, Comput. Phys. Commun., 14, 367

Brosius, J. W., Davila, J. M., Thomas, R. J., et al. 1997, ApJ, 477, 969

Brosius, J. W., Davila, J. M., Thomas, W. T., et al. 1993, ApJ, 411, 410

Cornille, M., Dubau, J., Mason, H. E., Blancard, C., \& Brown, W. A. 1997, A\&A, 320, 333
Dere, K. P. 1978, ApJ, 221, 1062

Dere, K. P., Landi, E., Young, P. R., \& Del Zanna, G. 2001, ApJS, 134, 331

Dyall, K. G., Grant, I. P., Johnson, C. T., Parpia, F. A., \& Plummer, E. P. 1989, Comput. Phys. Commun., 55, 424

Eissner, W., Galavis, M. E., Mendoza, C., \& Zeippen, C. J. 1999, A\&AS, 136, 385

Eissner, W., Jones, M., \& Nussbaumer, H. 1974, Comput. Phys. Commun., 8, 270

Eissner, W., \& Seaton, M. J. 1972, J. Phys. B, 5, 2187

Gu, M. F. 2003, ApJ, 582, 1241

Hibbert, A. 1975, Comput. Phys. Commun., 9, 141

Jonauskas, V., Keenan, F. P., Foord, M. E., et al. 2004, A\&A, 416, 383

Keenan, F. P., Conlon, E. S., Foster, V. J., et al. 1994, ApJ, 432, 809

McKeown, K., Aggarwal, K. M., Keenan, F. P., \& Rose, S. J. 2004, Phys. Scr., 70, 295

Neupert, W. M., Brosius, J. W., Thomas, R. J., et al. 1992, ApJ, 392, L95

Norrington, P. H., \& Grant, I. P. 2006, Comput. Phys. Commun., in preparation

Sampson, D. H., Zhang, H. L., \& Fontes, C. J. 1990, ADNDT, 44, 209 Sandlin, G. D., Brueckner, G. E., Scherrer, V. E., \& Tousey, R. 1976, ApJ, 205, L47

Tayal, S. S. 1994, ApJ, 426, 449

Thomas, R. J., \& Neupert, W. M. 1994, ApJS, 91, 461

Young, P. R., Landi, E., \& Thomas, R. J. 1998, A\&A, 329, 291 